\title{
Anti-NMDA Receptor Encephalitis in a Pregnant Woman
}

\section{Case Report}

Journal of Epilepsy Research pISSN 2233-6249 / eISSN 2233-6257

Received April 20, 2015

Accepted June 6, 2015

Corresponding author: Jiyoung Kim

Department of Neurology Pusan National

University Hospital, 179 Gudeok-ro,

Seo-gu, Busan 602-739, Korea

Tel. $+82-51-240-7311$

Fax. +82-51-254-7317

E-mail; bijoukim78@gmail.com

\section{Jiyoung Kim, Seung Ha Park, Yu Ri Jung, Soon Won Park, Dae Soo Jung}

Department of Neurology, Pusan National University Hospital and Pusan National University School of Medicine, and BioMedical Research Institute, Pusan National University Hospital, Busan, Korea

Anti N-methyl-D-aspartate (NMDA) receptor encephalitis is one of the most common types of autoimmune synaptic encephalitis. Anti-NMDA receptor encephalitis commonly occurs in young women with ovarian teratoma. It has variable clinical manifestations and treatment responses. Sometimes it is misdiagnosed as a psychiatric disorder or viral encephalitis. To the best of our knowledge, anti-NMDA receptor encephalitis is a rare condition in pregnant women. We report a case of anti-NMDA receptor encephalitis in a pregnant woman who presented with abnormal behavior, epileptic seizure, and hypoventilation. (2015;5:29-32)

Key words: NMDA receptor antibodies, Ovarian teratoma, Pregnancy, Outcome

\section{Introduction}

Anti-N-methyl-D-aspartate receptor (NMDAR) encephalitis was first reported in 2005 in four patients with ovarian teratoma. ${ }^{1}$ Many cases of anti-NMDAR encephalitis have been reported since the antigen (Ag) for the NMDAR Antibody (Ab) was confirmed in $2007 .^{2}$ It is a type of autoimmune synaptic encephalitis. While the pathophysiology is not completely understood, it is known that the NMDAR Ab is principally associated with the antibody against NR1 subunit of the NMDA receptors. ${ }^{3}$ The patient with anti-NMDAR encephalitis develops fever, headache, abnormal behavior, memory loss, autonomic dysfunction, seizure, hypoventilation, and dyskinesia. Sometimes the patient is misdiagnosed as having schizophrenia because of behavioral disturbance. ${ }^{4}$ Lim et al. reported that, in Korea, the most frequently presented symptoms of patients were psychiatric (68.8\%) followed by epileptic seizure $(50.0 \%) .{ }^{5}$ Although anti-NMDAR encephalitis can occur in females or males, it frequently occurs in women with ovarian teratoma. ${ }^{6}$ However, to the best of our knowledge, anti NMDAR encephalitis is a rare condition in pregnant women. We report a case of anti NMDAR encephalitis in a pregnant woman with ovarian teratoma who presented with abnormal behavior, hypoventilation, and epileptic seizure. Also we give a report long term clinical treatment outcome.

\section{Case}

A 28-year-old woman presented with abnormal behavior, hypo- ventilation, and epileptic seizure. At the time, she was pregnant (gestational age 7 weeks +4 days). She had mild fever, headache, and sleep disturbance for the previous two weeks. She had neither previous medical history nor medication. An emergency brain computed tomography (CT) scan, brain magnetic resonance imaging (MRI), and a lumbar puncture were performed while the patient was in the emergency room. Emergency CT scan and brain MRI were normal (Fig. 1A). Cerebrospinal fluid (CSF) analysis revealed pressure of $230 \mathrm{mmH}_{2} \mathrm{O}, 162$ white cells per $\mathrm{mm}^{3}$ (92\% lymphocytes), $48 \mathrm{mg} / \mathrm{dl}$ glucose, $46.2 \mathrm{mg} / \mathrm{dl}$ proteins with no organisms on the Gram stain, and negative CSF cultures. We initially considered her symptoms were indicating a viral encephalitis, and empiric therapy with acyclovir (10 mg/Kg/8 hr) was thus initiated. Transabdominal ultrasonography was performed to evaluate the fetal status in the emergency room. The fetus was healthy; however, we could not detect ovarian teratoma from the transabdominal ultrasonography.

After admission, the patient rapidly developed hypoventilation, epileptic seizure, and dyskinesia, and showed comatose mentality several days later. She was given an anti-epileptic drug (levetiracetam $1,500 \mathrm{mg} /$ day) and mechanical ventilation. During her stay in the intensive care unit (ICU), the fetus miscarried, the cause of which is unclear. After 2 weeks of admission, her autoimmune synaptic antibody test results were reported. Serum and CSF were positive for the anti-NMDAR Ab. We started intravenous methylprednisolone (1,000 $\mathrm{mg} /$ day) for 5 days and promptly initiated the 5 -day course of intravenous immunoglobulin ( $0.4 \mathrm{~g} / \mathrm{Kg} / \mathrm{day})$. After these treatments, we maintained a low dose of oral corticosteroids. We again performed 

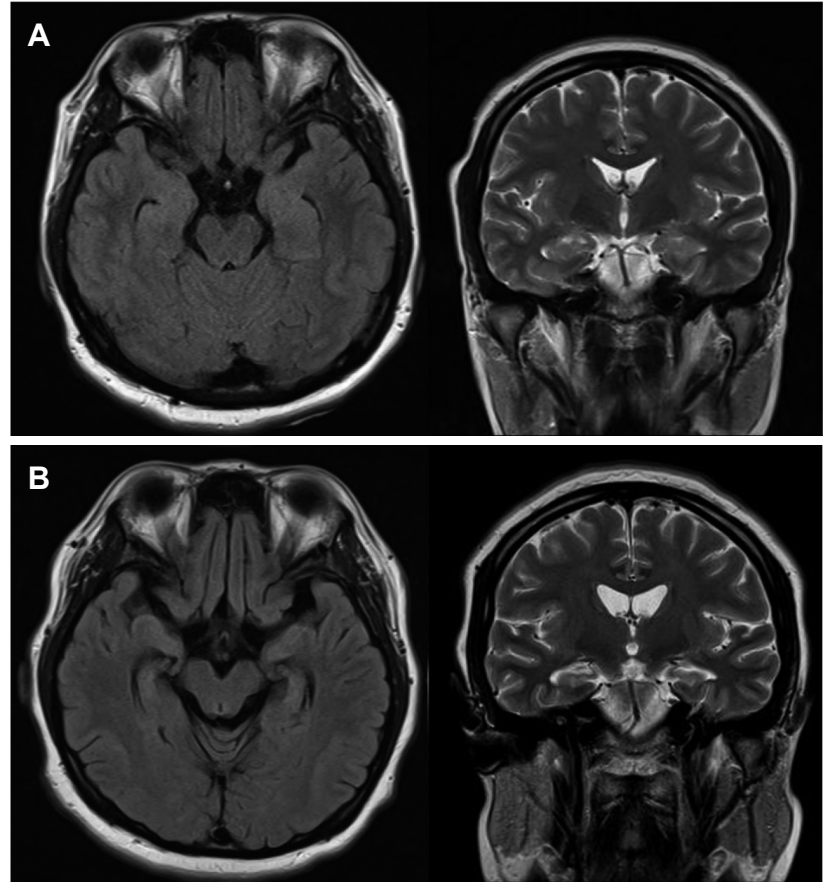

Figure 1. Brain MRI. (A) Initial brain MRI was normal. (B) Follow-up MRI, ten months after the onset of symptoms, shows diffuse brain atrophy and bilateral hippocampal atrophy compared with the initial brain MRI (A) on fluid attenuation inversion recovery (FLAIR) and T2 weighted images (T2WI).
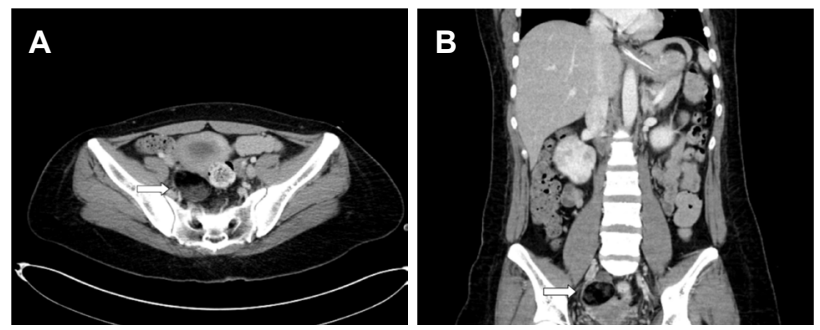

Figure 2. Abdomen-Pelvic $\mathrm{CT}$. (A) The axial abdomen-pelvic $\mathrm{CT}$ shows a large cystic mass $(4.8 \times 5.0 \mathrm{~cm}$, white arrow) in the right adnexa. (B) The coronal abdomen-pelvic CT shows a large cystic mass (white arrow), which was confirmed to be a mature cystic teratoma. transabdominal ultrasonography to detect the ovarian teratoma and informed the sonographer that she may have ovarian teratoma. However, we failed to detect the ovarian teratoma. We performed an abdomen-pelvic $\mathrm{CT}$ and it showed right ovarian teratoma (Figs. 2A, B). We removed the ovarian teratoma and performed plasmapheresis six times. However, her comatose mental state did not improve. Epileptic seizures were observed intermittently and ictal electroencephalogram (EEG) was developing from the left frontocentrotemporal area and spreading to the left hemisphere (Figs. 3A, B). Her symptom of hypoventilation persisted and a mechanical ventilator was used continuously. We considered using second-line therapy such as rituximab. However, pneumonia and cerebral salt wasting syndrome could not be controlled, causing a delay of several weeks in starting rituximab. After seven weeks, we started rituximab. Rituximab was administered every week, for 4 weeks $\left(375 \mathrm{mg} / \mathrm{m}^{2}\right)$. From one week after the start of rituximab therapy, her mental status and respiration were improved. From two weeks after the start of rituximab therapy, she was able to obey a one step command and breathe via self respiration without a mechanical ventilator. She was discharged to home after 22 weeks of hospital treatment (Fig. 4), and she regularly visited the outpatient neurological clinics. Follow-up brain MRI was performed at 10 months from the onset of her clinical symptoms. Brain MRI showed diffuse brain atrophy and bilateral hippocampal atrophy on fluid attenuation inversion recovery (FLAIR) images and T2 weighted images(T2WI). (Fig. 1B). However, there was continuous improvement in the cognitive function and the follow-up modified Rankin Scale (mRS) score was 1 at one year from the onset of her symptoms.

\section{A}

\section{B}
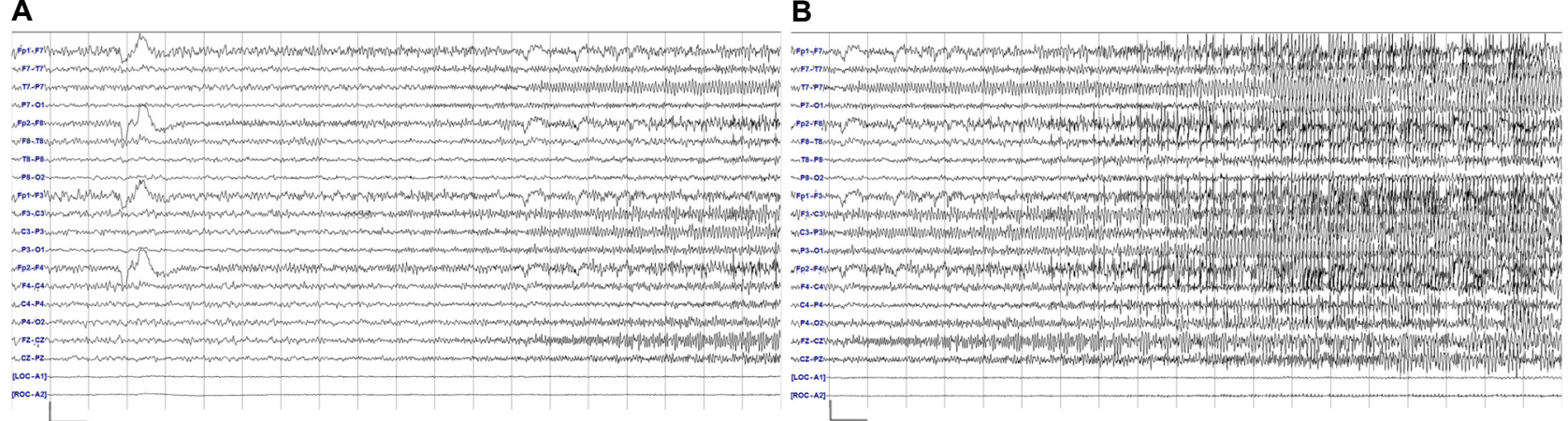

Figure 3. $E E G$ in ICU (A) Ictal EEG is developing from left frontocentrotemporal area. Her seizure was a tonic seizure during this recording. (B) Ictal EEG is spreading to the left hemisphere and obscured by muscle artifact (vertical scale: 100 uv, horizontal scale: 1 sec). 


\section{Clinical course}

\begin{tabular}{|l|l|}
\hline \multicolumn{1}{|c|}{$\begin{array}{l}\text { Comatose mentality } \\
\text {-Hypoventilation } \\
\text {-Epileptic seizure }\end{array}$} \\
\hline $\begin{array}{c}\text { •Abnormal } \\
\text { behavior }\end{array}$ & 'Worsened Pneumonia \\
\hline
\end{tabular}
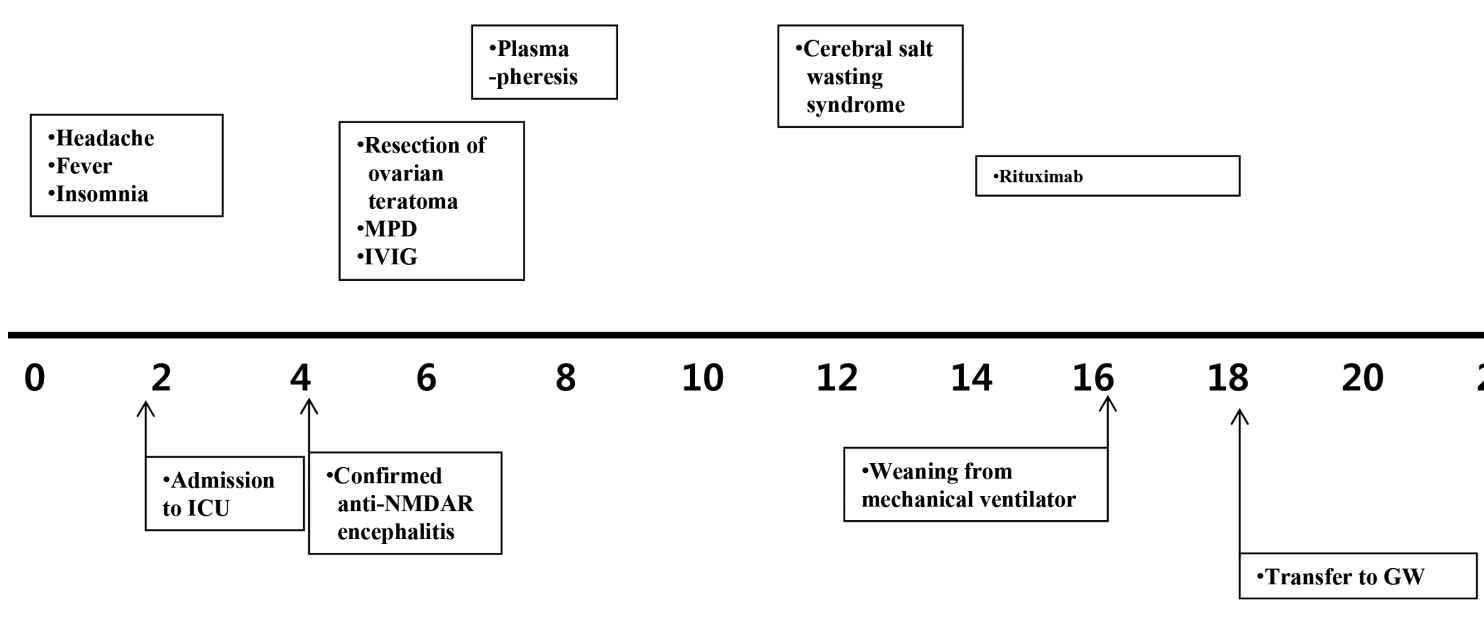

22 (weeks)

$\cdot \mathrm{mRS}$ scores

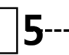

Figure 4. Clinical course and treatment. A patient presented comatose mentality, hypoventilation, and occasionally epileptic seizure in ICU and was treated with corticosteroid, IVIG, plasmaprhresis, and rituximab. ICU, intensive care unit; NMDAR, N-methyl-d-aspartate receptor MPD, methylprendnisolone; IVIG; intravenous immunoglobulin; GW, general ward; mRS, modified Rankin Scale.

\section{Discussion}

This is a case report of anti-NMDAR encephalitis in a pregnant woman. We performed transabdominal ultrasonography two times. The first transabdominal ultrasonography was performed to evaluate the fetal status. The second transabdominal ultrasonography was performed to detect the ovarian teratoma. However, we failed to detect the ovarian teratoma using transabdominal ultrasonography. According to research, variable accuracy of diagnostic value on ovarian teratoma using ultrasonogram has been reported. Mais et al. reported a sensitivity of $57.9 \%$ and specificity of $99.7 \%$ in diagnosing mature cystic teratoma using transvaginal ultrasonography. ${ }^{7}$ Patel et al. reported that the combination of transabdominal ultrasonogram and transvagianal ultrasonogram showed $85 \%$ sensitivity for the diagnosis and identification of cystic teratoma. ${ }^{8}$ Occasionally, ultrasonography is not sufficient to detect ovarian teratoma. In the emergency room, we could not actively use the radiological diagnostic tool because she was pregnant. Also, we did not conduct the abdominal-pelvic MRI in the emergency room. We considered her viral encephalitis and her symptoms had rapidly deteriorated. We did not have sufficient time to perform abdominal-pelvic MRI in the early period of the disease. After two weeks of admission, the NMDAR Ab was found to be positive, and we finally detected ovarian teratoma with the abdominal-pelvic $\mathrm{CT}$ after her fetus had miscarried. Late detection of ovarian teratoma delayed her treatment. However, although treatment required a long period of time, her mental status and cognition gradually improved and were nearly normal at the follow-up after one year from the onset of symptoms.

Anti-NMDAR encephalitis in pregnant women has been rarely reported. ${ }^{9,10}$ However, Anti-NMDAR encephalitis in pregnant women has recently been more frequently reported with growing concern about Anti-NMDAR encephalitis. Also, developmental outcomes have more frequently reported that fetuses and babies have been born from mothers with anti-NMDAR encephalitis. Chan et al. reported Anti-NMDAR encephalitis in a pregnant woman who miscarried within 2 days of hospitalization. ${ }^{11}$ In another case of Anti-NMDAR encephalitis in a pregnant woman, Jagota et al. reported that the baby showed NMDR antibody titer at the same level as that of the mother 2 days after birth and was negative at 1 year. ${ }^{12}$ Also, the baby showed developmental delay and cortical dysplasia in a brain MRI. It 
is unclear whether the causes of delayed development and cortical dysplasia are the transfer of maternal antibodies, materanal medication, or the indirect effect of maternal illness. Lamale-Smith et al. reported that NMDAR antibodies were capable of maternal-fetal transfer and reported appropriate development of a baby at one year. ${ }^{13}$ Kumar et al. reported three cases of anti-NMDAR encephalitis in pregnant women. ${ }^{14}$ These three patients developed symptoms between 8 and 17 weeks. In one of these patients, who had bilateral ovarian teratoma and severe neurological complications, the pregnancy was terminated. However, two of these patients gave birth to normal babies. The authors suggested the good outcome of these two neonates was related to the period of transplacental transfer of Immunoglobulin $\mathrm{G}(\mathrm{lgG})$. Ig $\mathrm{g} 1$ and $\mathrm{G} 3$ cross the placenta by binding to Fc neonatal receptor present in the syncytiotrophoblast and this process of placental transfer occurs at around 14 to 16 weeks. ${ }^{15}$ This results in a very low level of fetal blood IgG at the time of onset of clinical symptoms. Additionally, the fetal blood-brain barrier becomes functional by the end of the second trimester, and this is one of the reasons for the delivery of a normal baby.

In summary, this case highlighted the importance of active attempts to detect the ovarian teratoma in pregnant women with anti-NMDAR encephalitis. If pregnancy limits the use of a radiologic diagnostic tool such as abdomen-pelvic CT, an abdominal-pelvic MRI should be performed as early as possible. Transabdominal or transvaginal ultrasonography is not sufficient for detecting ovarian teratoma in some cases. Also, various treatments should be performed. A long time was needed to recover from the comatose mentality and restore cognition. Lastly, the clinician should be concerned about the best way to determine a fetal developmental outcome. Later anti-NMDAR encephalitis in cases of pregnant women may resolve this issue.

\section{References}

1. Vitaliani R, Mason W, Ances B, Zwerdling T, Jiang Z, Dalmau J. Paraneoplastic encephalitis, psychiatric symptoms, and hypoventilation in ovarian teratoma. Ann Neurol 2005;58:594-604.

2. Dalmau J, Tuzun E, Wu HY, et al. Paraneoplastic anti-N-methyl-D-aspartate receptor encephalitis associated with ovarian teratoma. Ann Neurol 2007;61:25-36.

3. Dalmau J, Lancaster E, Martinez-Hernandez E, Rosenfeld MR, BaliceGordon R. Clinical experience and laboratory investigations in patients with anti-NMDAR encephalitis. Lancet Neurol 2011;10:63-74.

4. Lee HS, Kim SW, Chung SJ, Yoo HS, Lee PH, Choi SA. Anti-NMDA receptor encephalitis which has shown clinical symptoms of schizophrenia. J Korean Neurol Assoc 2013;31:115-7.

5. Lim JA, Lee ST, Jung $\mathrm{KH}$, et al. Anti-N-methyl-d-aspartate receptor encephalitis in Korea: clinical features, treatment, and outcome. J Clin Neurol 2014;10:157-61.

6. Titulaer MJ, McCracken L, Gabilondo I, et al. Treatment and prognostic factors for long-term outcome in patients with anti-NMDA receptor encephalitis: an observational cohort study. Lancet Neurol 2013;12: 157-65.

7. Mais V, Guerriero S, Ajossa S, Angiolucci M, Paoletti AM, Melis GB. Transvaginal ultrasonography in the diagnosis of cystic teratoma. Obstet Gynecol 1995;85:48-52.

8. Patel MD, Feldstein VA, Lipson SD, Chen DC, Filly RA. Cystic teratomas of the ovary: diagnostic value of sonography. $A / R$ Am J Roentgenol 1998; 171:1061-5.

9. McCarthy A, Dineen J, McKenna $P$, et al. Anti-NMDA receptor encephalitis with associated catatonia during pregnancy. I Neurol 2012;259: 2632-5.

10. Ito $Y$, Abe T, Tomioka R, Komori T, Araki N. [Anti-NMDA receptor encephalitis during pregnancy]. Rinsho Shinkeigaku 2010;50:103-7.

11. Chan LW, Nilsson C, Schepel J, Lynch C. A rare case of anti-N-methyl-D-aspartate receptor encephalitis during pregnancy. N Z Med J 2015;128:89-91.

12. Jagota P, Vincent A, Bhidayasiri R. Transplacental transfer of NMDA receptor antibodies in an infant with cortical dysplasia. Neurology 2014; 82:1662-3.

13. Lamale-Smith LM, Moore GS, Guntupalli SR, Scott JB. Maternal-fetal transfer of anti-N-methyl-d-aspartate receptor antibodies. Obstet Gynecol 2015; 125:1056-8.

14. Kumar MA, Jain A, Dechant VE, et al. Anti-N-methyl-D-aspartate receptor encephalitis during pregnancy. Arch Neurol 2010;67:884-7.

15. Roopenian DC, Akilesh S. FcRn: the neonatal Fc receptor comes of age. Nat Rev Immunol 2007;7:715-25. 